\title{
BACKGROUND HEATFLOW ON HOTSPOT PLANETS: IO AND VENUS
}

\author{
David J. Stevenson and Sean C. McNamara
}

Division of Geological and Planetary Sciences, California Institute of Technology

\begin{abstract}
On planets where most of the heat is transported to the surface by igneous activity (extrusive volcanism or near-surface intrusions), the surface heatflow at localities well away from regions of current igneous activity need not be even approximately the conductive heatflow through the entire lithosphere but may instead be dominated by the residual heat leaking out from the last igneous event in that locality. On Io, it is likely that $(\kappa \tau)^{1 / 2} \ll$ lithosphere thickness $(\kappa=$ thermal diffusivity, $\tau=$ typical time between "resurfacing" events) and the background heatflow may be very large, comparable or even larger than the current observational heatflow, which is associated with the hotspots alone. This upward revision of Io's heatflow is compatible with observations and with recent indications of a non-steady tidal and thermal evolution. On Venus, $(\kappa \tau)^{1 / 2}$ is probably comparable to the lithosphere thickness and the resulting upward revision of heatflow may be only marginally significant, unless magmatic activity is enormously greater than on Earth.
\end{abstract}

\section{Introduction}

On a solid planet, the simplest view of the surface heatflow is expressed by the equation

$$
F=\frac{k \Delta T}{d_{T}}
$$

where $F$ is the heat flux, $k$ is the thermal conductivity, and $\Delta T$ is the temperature drop across the thermal lithosphere of thickness $d_{T}$. This is approximately correct on Earth, despite the delivery of some heat by volcanism and hydrothermal circulation. Lithospheric radiogenic heating changes the result by at most a factor of two. It is believed to also apply for relatively low heatflow planets such as Mercury, Moon, and Mars. It has become increasingly apparent, however, that it need not apply on high heatflow bodies where hotspot volcanism dominates. Io is a striking example of a body where the topography is substantial, implying a thick lithosphere [Schaber, 1982; Nash et al., 1986; Carr, 1986] and the heatflow is greater than about $1400 \mathrm{erg} \mathrm{cm}^{-2} \mathrm{~s}^{-1}$ [Johnson et al., 1984], clearly incompatible with equation (1). In a deceptively simple but important paper, O'Reilly and Davies [1981] pointed out that Io delivers its heat through heat pipes to the surface, effectively decoupling the thickness of the lithosphere from the magnitude of the heat flow. A similar though less extreme application to Venus has been proposed by Turcotte [1988].

Although there is increasing acceptance of the failure of equation (1) for hotspot planets, there is still the common assumption (sometimes explicit, often implicit) that the heatflow away from regions of current volcanic activity (hereafter called the background heatflow) is conductive and expressed by equation (1). Many papers discussing

Copyright 1988 by the American Geophysical Union.

Paper number 88GL04023.

0094-8276/88/88GL-04023\$03.00 the global heatflow of Io have expressed the view that the total background heatflow is less (perhaps much less) than the hotspot component (e.g., Gaskell et al. [1988]). The purpose of this paper is to challenge this assumption. The potential fallacy in the assumption is easily understood: Consider a planet for which the volcanic activity is large enough that every point on the surface has been within a volcanically active region many times in the past. If the recurrence time $\tau$ (the time between epochs of activity at any location) is short enough that $(\kappa \tau)^{1 / 2}<d_{T}$, where $\kappa$ is the thermal diffusivity, and if the amount of volcanic material is sufficient then the background heatflow at any location will be dominated by the residual heat of the last volcanic event rather than by heat conducted through the entire lithosphere. From this point of view, the thickness of the lithosphere is determined by difficult issues such as the sustainable length of volcanic conduits and the mechanics of magmafracturing, rather than by considerations of thermal conduction. It is argued here that Io is probably in the regime $(\kappa \tau)^{1 / 2} \ll d_{T}$ and has high enough resurfacing rates that the background heatflow may be very large, comparable or larger than the hotspot component (in the way it is defined observationally). This is compatible with recent orbital evidence for non-steady state evolution of Io. On Venus, it is conceivable that $d_{T} \gtrsim(\kappa \tau)^{1 / 2}$; this might reduce estimates for the required number of currently active hotspot centers, but only if the magmatic activity on Venus is enormously greater than on Earth. As discussed below, this is unlikely.

\section{The Model}

Consider first, an emplacement of a layer of hot material of thickness $d$ and temperature excess $\Delta T$ over a cold surface (defined to have $T=0$ ). Neglecting latent heat, the equation and initial boundary conditions for conductive cooling are

$$
\begin{aligned}
& \frac{\partial T}{\partial t}=\kappa \frac{\partial^{2} T}{\partial z^{2}} \\
T(0, t) & =0 \\
T(z, 0) & =\Delta T \quad 0<z<d \\
& =0 \quad z>d
\end{aligned}
$$

for which the solution is [Landau and Lifshitz, 1959]

$$
\begin{aligned}
T(z, t)=\Delta T & \{\operatorname{erf}[(x-d) / 2 \sqrt{\kappa t}] \\
& -\operatorname{erf}[(x+d) / 2 \sqrt{\kappa t}]\}
\end{aligned}
$$

The heat flux corresponding to this is

$$
F=\frac{k \Delta T}{\sqrt{\pi \kappa t}}\left(1-e^{-d^{2} / 4 \kappa t}\right)
$$

As one would intuitively expect, $F \propto t^{-1 / 2}$ (like a cooling half space) for $t \lesssim d^{2} / 4 \kappa$ and $F \propto t^{-3 / 2}$ once the entire interior of the flow is losing heat to the surface and below. 
This exact solution does not quite suit our purpose because it is a single event. Consider a model in which resurfacing occurs by emplacing a layer of thickness $d$ at times $\tau, 2 \tau, 3 \tau, \cdots$. We carried out a number of numerical solutions of the diffusion equation under these circumstances. For $d^{2} \ll 4 \kappa \tau$ we found, not surprisingly, that little memory is retained of previous flows and the behavior is well described by eq. (4) with $t$ measured relative to the time of last emplacement. If $d^{2} \gg 4 \kappa \tau$ then the flows do not cool internally before the next flow is emplaced. In this limit, no lithosphere can exist. In fact, this is not a meaningful limit for our purposes (it might, however, describe the development of a magma ocean). The most difficult case is the regime where $d^{2} / \kappa \tau$ is not enormously different from unity. This might seem like a special (hence implausible) regime, but it may even be a preferred regime if the eruption process proceeds once the overlying layer has cooled enough to allow fracture. In this regime, an approximate solution can be obtained, by allowing for a fraction $\epsilon$ of the heat to be retained from each resurfacing event. This suggests an expression for the heat flux of the form

$$
F \simeq \frac{k \Delta T}{\sqrt{\pi \kappa t}}\left[1-(1-\epsilon) e^{-d^{2} / 4 \kappa t}\right]
$$

where the value of $\epsilon$ was estimated from numerical simulations such as that shown in Figure 1, and is given roughly by

$$
\begin{gathered}
\epsilon \simeq 0.2 \frac{d^{2}}{\kappa \tau} \quad \tau \gtrsim d^{2} / \kappa \\
\epsilon \simeq 1-0.8\left(\frac{\kappa \tau}{d^{2}}\right)^{1 / 2} \quad, \quad \tau \lesssim d^{2} / \kappa
\end{gathered}
$$

By conservation of energy, the time averaged heat flux at any locality is

$$
\begin{gathered}
\bar{F} \simeq \rho C_{p} \Delta T u(1-\epsilon) \\
u \equiv d / \tau
\end{gathered}
$$

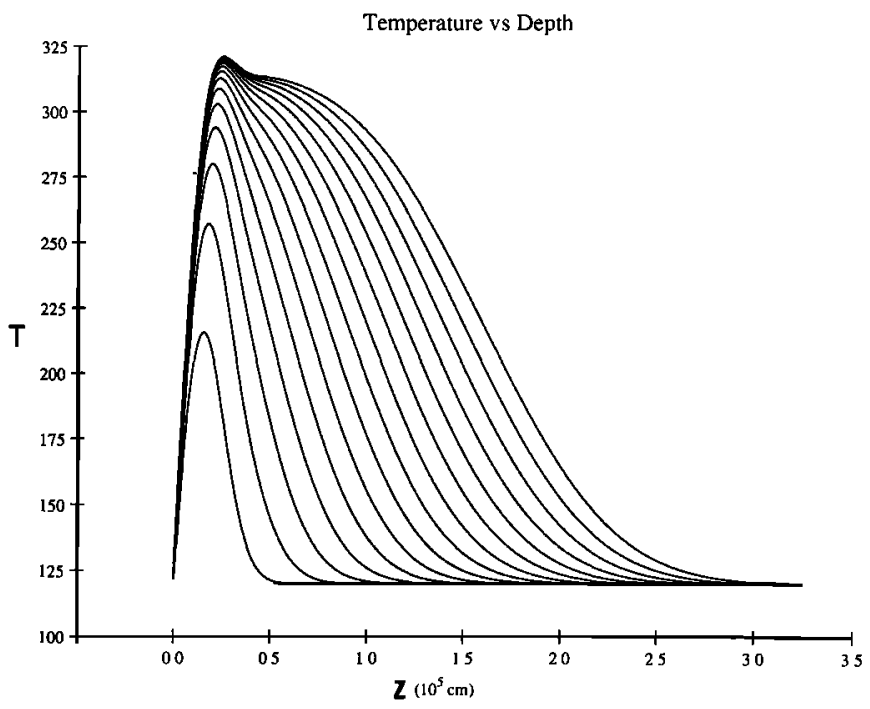

Figure 1. A sequence of numerically computed temperature profiles at successive times $(\tau, 2 \tau, 3 \tau, \cdots)$ just prior to a resurfacing event, for the particular case $d^{2}=\kappa \tau$ and an equilibrium surface temperature appropriate to Io. In each case, zero on the horizontal axis refers to the actual surface, so the residual (unescaped) heat is trapped deeper down after each new resurfacing.

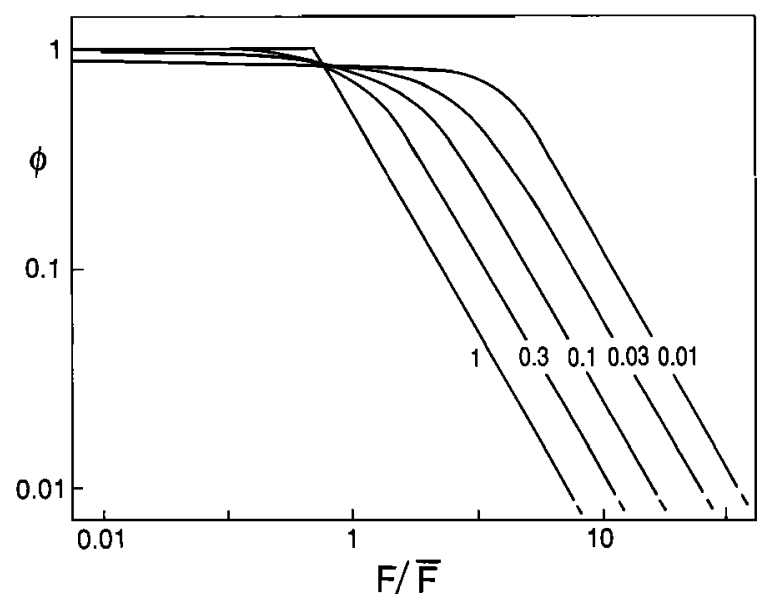

Figure 2. Fraction $(\phi)$ of total heat flow that comes from the regions where the heat flow exceeds a value $F$ (nondimensionalized by the mean heatflow $\bar{F}$ ). Each curve is labeled by the value of $\mathrm{Pe}$, the Peclet number (see text). At high Pe, most of the heatflow comes from low heatflow regions.

where $u$ is the resurfacing rate. The requirement that this is large compared to the conductive heat flux through the lithosphere is

$$
u(1-\epsilon) d_{T} / \kappa \gg 1
$$

The requirement that a lithosphere exist is $\epsilon \lesssim 1 / 2$ or $u d / \kappa \Sigma 1$. It is easy to satisfy this simultaneously with equation (7) if $d \ll d_{T}$. All these equations omit latent heat effects but these are only a 10-20\% correction, less important than the other uncertainties in the model.

From the point of view of observations, it is much more interesting to ask how the heat flow is distributed on the surface, since this may provide an observational constraint. Consider, first, the "boxcar" model

$$
P(t)=\frac{1}{\tau} \quad, \quad 0<t<\tau
$$

where $P$ is the probability that any given point on the surface underwent resurfacing at a time $t$ before the present. For the simple "optimal" case of $F \simeq$ $k \Delta T / \sqrt{\pi \kappa t}$ and $\tau \sim d^{2} / 2 \kappa$, this model predicts that the normalized probability of a given heat flux $F$ is

$$
\begin{aligned}
& P(F) \simeq 2 F_{\text {min }}^{2} / F^{3}, \quad F_{\text {min }} \leq F \\
& F_{\text {min }} \equiv k \Delta T / \sqrt{\pi \kappa \tau}
\end{aligned}
$$

The fraction of the surface at high heatflow is small. For example, only $1 \%$ of the surface has heatflows in excess of $10 F_{\text {min }}$. The fraction of heat output from regions with heatflow greater than $F$ is

$$
\phi(F) \equiv \int_{F}^{\infty} F P(F) d F / \int_{F_{u<\ln }}^{\infty} F P(F) d F
$$

and has the value $F_{\text {uin }} / F$ in this particular case. Half of the heat output occurs from regions that have heatflows less than the mean heatflow $\left(2 F_{\text {min }}\right)$. This has important implications for the (non)detectability of the background heatflow on Io. In Figure $2, \phi(F)$ is shown for a variety of models. If the heatflow is non-dimensionalized by its mean value then the only remaining variable is a Peclet number 


$$
\mathrm{Pe} \equiv \frac{d^{2}}{2 \kappa \tau}=\frac{u d}{2 \kappa}
$$

which can be best interpreted as the fractional time of high heatflow (i.e., the ratio of thermal diffusion time for the resurfacing layer to the recurrence time of volcanic events at any given locality). The factor of two in the definition improves this convenient interpretation. The simple example above (eq. (10)) corresponds to the maximal value, $\mathrm{Pe} \simeq 1$. As $\mathrm{Pe}$ decreases, a larger fraction of the heat output occurs from regions with higher heatflows, but there is always a substantial low heatflow component even at low Pe.

\section{Application to Io}

The anomalous infrared emission properties of Io have long been known but were not correctly interpreted until after the discovery of volcanism during the Voyager 1 flyby. The analysis of both ground-based and Voyager data has led to the unequivocal identification of an internal heatflow of at least $1400 \mathrm{erg} \mathrm{cm} \mathrm{cm}^{-2} \mathrm{~s}^{-1}$ and possibly more [Matson et al., 1981; Johnson et al., 1984; Nash et al., 1986; Johnson et al., 1988]. The identification is unequivocal not so much because of the magnitude of this heatflow (which is only about $10 \%$ of the absorbed insolation) but because it is associated with temperatures (ranging from $200 \mathrm{~K}$ upward) that are high compared with typical surface temperatures. In other words, the "spectral contrast" (the steep rise of brightness temperature with decreasing wavelength) is an essential part of identifying this heatflow. In the context of the models presented above, we must ask: What is the spectral contrast of the background heatflow? The brightness temperature at each wavelength can be evaluated from

$$
B\left(\lambda, T_{b}\right)=\int_{0}^{\infty} f(T) B(\lambda, T) d T
$$

where $B(\lambda, T)$ is the blackbody emission (Planck function) at wavelength $\lambda, T_{b}$ is the brightness temperature, and $f(T)$ is the probability that a surface element of Io lies in the temperature range between $T, T+d T$ :

$$
f(T)=\frac{-P(t) d F / d T}{d F / d t}
$$

with the heatflux $F$ given by equation (5). We also express

$$
F=\sigma\left(T^{4}-T_{o}^{4}\right)
$$

where $\sigma$ is the Stefan-Boltzmann constant and $T_{o}$ is the insolation temperature. Our purpose here is not to model Io specifically (since that requires detailed assumptions about surface properties) but to show how the spectral contrast varies with total heatflow and Peclet number. This is illustrated in Figure 3 , for the choice $T_{o}=120 \mathrm{~K}$ and two background mean heatflows (1500 and 3000 erg $\mathrm{cm}^{-2} \mathrm{~s}^{-1}$ ). As these calculations show, a large background heatflow is possible without substantial spectral contrast, provided $\mathrm{Pe}$ is quite large. These heatflows correspond to mean resurfacing rates of up to a few $\mathrm{cm} / \mathrm{yr}$ [Johnson and Soderblom, 1982; Carr, 1986] and imply that the interior of Io is only partially molten [Webb and Stevenson, 1987].

It is difficult to make independent estimates of plausible values for $\mathrm{Pe}$, the ratio of cooling time for a resurfacing event to the time between resurfacing events, espe-

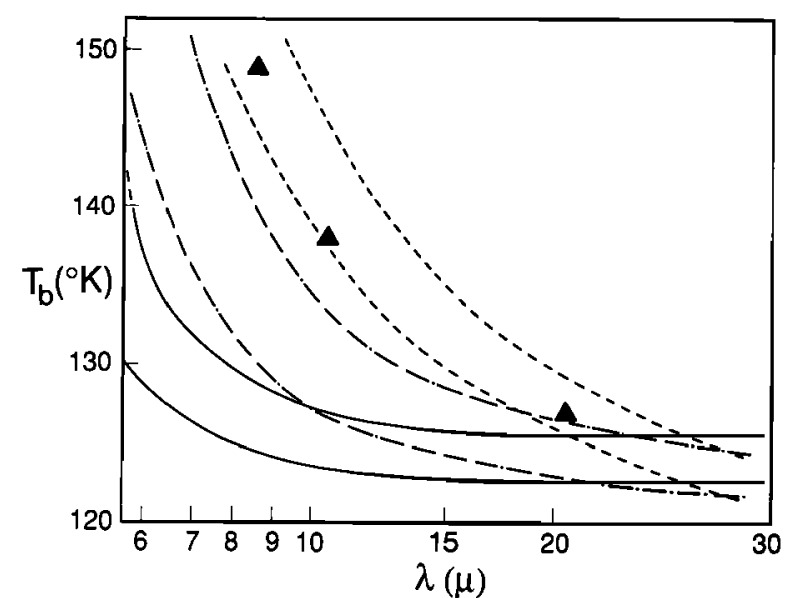

Figure 3. Brightness temperature $T_{b}$ as a function of wavelength for three sets of models: $-(\mathrm{Pe}=1)$, $-1 \mathrm{Pe}=0.1),--(\mathrm{Pe}=0.01)$. In each case, the lower curve corresponds to a mean heatflow of 1500 erg $\mathrm{cm}^{-2} \mathrm{~s}^{-1}$ and the upper curve corresponds to 3000 erg $\mathrm{cm}^{-2} \mathrm{~s}^{-1}$. The Io data are triangles at $8.4,10.6$, and $21 \mu$ (Matson et al. [1981]); the curves are not meant to fit the data (but should lie below them).

cially when allowance is made for near surface intrusive events. Certainly, $\mathrm{Pe}$ is at least as large as the fractional surface of Io that is currently active, assuming that Io is in a typical state at present. (This is a debatable assumption, but probably not wrong by more than a factor of a few; see below.) This suggests $\mathrm{Pe} Z 10^{-2}$. However, a substantially larger value would seem plausible. For example, a volcanic province that erupts every $10^{4}$ years and lays down three hundred meters of material at each eruption would have $\mathrm{Pe} \sim 0.3$, assuming $K \simeq 10^{-2}$ $\mathrm{cm}^{2} \mathrm{~s}^{-1}$. If the actual eruption (i.e., period of activity) takes $\sim 10^{2}$ years and all parts of the surface of lo are equally suitable for eruptions then the "active fraction" of the surface would then be $\sim 10^{-2}$, much less than the value of Pe. Figure 3 then implies that the spectral contrast is low and the globally averaged background heatflow could be large; comparable or even larger than the hotspot component.

Recent analysis of the coupled thermal and orbital evolution of Io are consistent with this possibility. If the total average heat output were less than $\sim 2000 \mathrm{erg} \mathrm{cm}^{-2} \mathrm{~s}^{-1}$ then it could be argued (e.g., Schubert et al. [1986]) that Io is in steady state, with its orbit steadily expanding at a rate given by Jupiter's tidal $Q$ and its orbital eccentricity determined by the ratio of Io's $Q$ to that of Jupiter [Yoder and Peale, 1981]. However, data on the expansion of Io's orbit suggest a much slower expansion than expected for the steady state model [Lieske, 1987; Greenberg, 1987] and accordingly allow for a higher heatflow, similar to (but not necessarily exactly compatible with) the non-steady state model of Ojakangas and Stevenson [1986]. The higher heatflow arises if the eccentricity of lo's orbit is currently decreasing. A global heat output of $3000-4000 \mathrm{erg} \mathrm{cm}^{-2} \mathrm{~s}^{-1}$ is possible. These arguments are only suggestive and the true test is observational.

\section{Application to Venus}

The current uncertain state of the interpretation of Venus geology [Basilevsky and Head, 1988] renders any statements about Venus conditional at best. If the total 
magmatic activity of Venus is not much different from Earth, as several have argued [Solomon and Head, 1982; Morgan and Phillips, 1983; Phillips and Malin, 1984], then the considerations developed above have marginal relevance. Specifically, consider a recurrence interval of $10^{8}$ years for magmatic activity, with $10 \mathrm{~km}$ of material (basaltic crust or gabbroic intrusion) "laid down." This corresponds to $\mathrm{Pe} \sim 0.03$ and $\lesssim 10 \%$ enhancement of the background heatflow. On the basis of crater counts [Barsukov et al., 1986; Grimm and Solomon, 1987], this recurrence interval is about the smallest conceivable. However, it must be stressed that intrusive activity can come close to achieving much of the same effect (in terms of heatflow) as surface lava flows, yet have a less direct impact on geomorphology, so the situation is not fully determined from the radar data. Turcotte [1988] has suggested that the magmatic activity could be two orders of magnitude higher on Venus than on Earth. Under these circumstances, the "background" heatflow will have comparable contributions from conduction through the lithosphere and cooling of previous igneous events, if the lithosphere thickness $d_{T} \sim 100 \mathrm{~km}$.

\section{Concluding Comments}

The main message of this paper is a rather obvious one, but one that seems to have been insufficiently appreciated: On planets where volcanism dominates the heatflow, there is no simple relationship between lithospheric thickness and heatflow, either locally or globally, even away from regions of current volcanic activity. There is no basis, either observationally or theoretically, to advocate the minimalist view that the Io heatflow is as low as the hotspot component alone would suggest. Our developing views of Io and (to a lesser extent) Venus will require a better understanding of how volcanism operates on these bodies.

Acknowledgements. Discussions with T. V. Johnson and D. L. Matson, and comments from a reviewer were helpful. Contribution number 4657 from the Division of Geological and Planetary Sciences, California Institute of Technology, Pasadena, California 91125. This work is supported by the NASA Planetary Geophysics program, grant NAGW-185.

\section{References}

Barsukov, U.L., et al., The geology and geomorphology of the Venus surface as revealed from the radar images obtained by Veneras 15 and 16, J. Geophys. Res. 91, D378-D398, 1986.

Basilevsky, A. T. and J. W. Head III, The geology of Venus, Ann. Rev. Earth Planet. Sci. 16, 295-317, 1988.

Carr, M. H., Silicate volcanism on Io, J. Geophys. Res. 91, 3521-3532, 1986.

Gaskell, R. W., S. P. Synnott, A. S. McEwen, and G. G. Schaber, Large scale topography of Io: Implications for internal structure and heat transfer, Geophys. Res. Lett. 15, 581-584, 1988.

Greenberg, R. Time-varying orbits and tidal heating of the Galilean satellites. International Workshop on Time-Variable Phenomena in the Jovian System, Flagstaff, Arizona, 1987.

Grimm, R.E. and S.C. Solomon, Limits on modes of lithospheric heat transport on Venus from impact crater densities, Geophys. Res. Lett. 14, 530-541, 1987.

Johnson, T. V., D. Morrison, D. L. Matson, G. J. Veeder, R. H. Brown, and R. M. Nelson, Io volcanic hotspots: Stability and longitudinal distribution, Science 226, 134-137, 1984.

Johnson, T. V., G. J. Veeder, D. L. Matson, R. H. Brown, R. M. Nelson, and D. Morrison, Io: Evidence for silicate volcanism in 1986, Submitted to Science, 1988.

Johnson, T. V. and L. A. Soderblom, Volcanic eruptions on Io: Implications for surface evolution and mass loss, In Satellites of Jupiter, D. Morrison, (ed.), pp. 634646, Univ. of Arizona Press, Tucson, 1982.

Landau, L. D. and E. M. Lifshitz, Fluid Mechanics, Addison-Wesley, Reading, Mass., p. 199, 1959.

Lieske, J. H., Galilean satellite evolution: Observational evidence for secular changes in mean motions, Astron. Astrophys. 176, 146-158, 1987.

Matson, D. L., G. A. Ransford, and T. V. Johnson, Heat flow from Io (J1), J. Geophys. Res. 86, 1664-1672, 1981.

Morgen, P. and R.J. Phillips, Hot spot heat transfer: Its application to Venus and implications to Venus and Earth, J. Geophys. Res. 88, 8305-8317, 1983.

Nash, D. B., M. H. Carr, J. Gradie, D. M. Hunten, and C. F. Yoder, Io, In Satellites, J. Burns and M. Matthews, (eds.), pp. 629-688, Univ. of Arizona Press, Tucson, 1986 .

O'Reilly, T. C. and G. F. Davies, Magma heat transport on Io: A mechanism allowing a thick lithosphere, Geophys. Res. Lett. 8, 313-316, 1981.

Ojakangas, G. W. and D. J. Stevenson, Episodic volcanism of tidally heated satellites with application to Io, Icarus 66, 341-358, 1986.

Phillips, R.J. and M.C. Malin, Tectonics of Venus, Ann. Rev. Earth Planet. Sci. 12, 411-443, 1984.

Schaber, G. G., The geology of Io, In Satellites of Jupiter, D. Morrison, (ed.), pp. 556-597, Univ. of Arizona Press, Tucson, 1982.

Schubert, G., T. Spohn, and R. T. Reynolds, Thermal histories, compositions and internal structures of the moons of the solar system, In Satellites, J. Burns and M. Matthews, (eds.), pp. 224-292, Univ. of Arizona Press, Tucson, 1986.

Solomon, S.C. and J.W. Head, Mechanisms for lithospheric heat transfer on Venus: Implications for tectonic style and volcanism, J. Geophys. Res. 87, 92369246, 1982.

Turcotte, D. L., A heat-pipe mechanism for volcanism and tectonics on Venus. LPSC XIX Abstracts 12071208 , and submitted to J. Geophys. Res., (1988).

Webb, E. K. and D. J. Stevenson, Subsidence of topography on Io, Icarus 70, 348-353, 1987.

Yoder, C. F. and S. J. Peale, The tides of Io, Icarus 47, $1-35,1981$.

S. C. McNamara and D. J. Stevenson, Division of Geological and Planetary Sciences, California Institute of Technology, Pasadena, California 91125.

(Received June 20, 1988;

revised October 24, 1988; accepted October 26, 1988.) 